University of Wollongong

Research Online

Faculty of Engineering and Information

Faculty of Engineering and Information

Sciences - Papers: Part A

Sciences

$1-1-2013$

Exact travelling wave solutions for some important nonlinear physical models

Jonu Lee

University of Wollongong

Rathinasamy Sakthivel

Sungkyunkwan University

Follow this and additional works at: https://ro.uow.edu.au/eispapers

Part of the Engineering Commons, and the Science and Technology Studies Commons

Research Online is the open access institutional repository for the University of Wollongong. For further information contact the UOW Library: research-pubs@uow.edu.au 


\title{
Exact travelling wave solutions for some important nonlinear physical models
}

\author{
Abstract \\ The two-dimensional nonlinear physical models and coupled nonlinear systems such as Maccari \\ equations, Higgs equations and Schrodinger-KdV equations have been widely applied in many branches \\ of physics. So, finding exact travelling wave solutions of such equations are very helpful in the theories \\ and numerical studies. In this paper, the Kudryashov method is used to seek exact travelling wave \\ solutions of such physical models. Further, three-dimensional plots of some of the solutions are also \\ given to visualize the dynamics of the equations. The results reveal that the method is a very effective and \\ powerful tool for solving nonlinear partial differential equations arising in mathematical physics.

\section{Keywords} \\ important, solutions, wave, travelling, models, exact, physical, nonlinear \\ Disciplines \\ Engineering | Science and Technology Studies

\section{Publication Details} \\ Lee, J. \& Sakthivel, R. (2013). Exact travelling wave solutions for some important nonlinear physical \\ models. Pramana: journal of physics, 80 (5), 757-769.
}




\begin{tabular}{|c|}
\hline $\begin{array}{r}\text { PRAMANA } \\
- \text { journal of } \\
\text { physics }\end{array}$ \\
\hline
\end{tabular}

\title{
Exact travelling wave solutions for some important nonlinear physical models
}

\author{
JONU LEE ${ }^{1}$ and RATHINASAMY SAKTHIVEL ${ }^{2, *}$ \\ ${ }^{1}$ School of Mathematics and Applied Statistics, University of Wollongong, Wollongong, \\ NSW 2522, Australia \\ ${ }^{2}$ Department of Mathematics, Sungkyunkwan University, Suwon 440-746, South Korea \\ *Corresponding author. E-mail: krsakthivel@yahoo.com
}

MS received 27 April 2012; revised 27 October 2012; accepted 16 November 2012

\begin{abstract}
The two-dimensional nonlinear physical models and coupled nonlinear systems such as Maccari equations, Higgs equations and Schrödinger-KdV equations have been widely applied in many branches of physics. So, finding exact travelling wave solutions of such equations are very helpful in the theories and numerical studies. In this paper, the Kudryashov method is used to seek exact travelling wave solutions of such physical models. Further, three-dimensional plots of some of the solutions are also given to visualize the dynamics of the equations. The results reveal that the method is a very effective and powerful tool for solving nonlinear partial differential equations arising in mathematical physics.
\end{abstract}

Keywords. Exact travelling wave solutions; nonlinear physical models; Kudryashov method.

PACS Nos $\quad$ 02.30.Jr; 02.70.Wz; 04.20.Jb

\section{Introduction}

The study of nonlinear partial differential equations is an active area of research in applied mathematics, theoretical physics and engineering fields. In particular, there has been considerable interest in seeking exact travelling wave solutions of nonlinear evolution equations that describe some important physical and dynamic processes. In the past several decades, many powerful methods such as variational iteration method [1], homotopy analysis method [2], homotopy perturbation technique [3], modified tanh-coth method [4], the Jacobi elliptic function method [5], $\left(G^{\prime} / G\right)$-expansion method [6-8], the expfunction method [9-12], trial equation method [13], spectral collocation method [14] and many other techniques were used to obtain exact travelling wave solutions of nonlinear problems. More precisely, there is no unified method that can be used to handle all types of nonlinear problems. A powerful and effective method for finding exact solutions of nonlinear differential equations was proposed in [15]. In particular, this method allows 
us to obtain all solitary wave solutions and all one-periodic solutions when we get the expansion of the general solution of nonlinear differential equation in the Laurent series. Moreover, the main advantage of this method is that we can construct exact solutions of high order nonlinear evolution equations more effectively in comparison with other methods. In [16-18], the author applied this method to construct the exact solutions of some nonlinear non-integrable equations. Ryabov [19] obtained exact solutions of the Kudryashov-Sinelshchikov equation by using the Kudryashov method. Kabir [20] used the modified Kudryashov method to construct the solitary travelling wave solutions of the Kuramoto-Sivashinsky and seventh-order Sawada-Kotera equations.

Explicit solutions to nonlinear problems are of fundamental importance. The travelling wave solutions may be useful in the theoretical and numerical studies of the model systems. Therefore, finding travelling wave solutions of nonlinear equations is of fundamental interest to understand the equations fully. In this paper, we shall find the exact travelling wave solutions for some nonlinear physical models and coupled equations such as Higgs equation, Maccari system and Schrödinger-KdV equation [6,7] by using the Kudryashov method. The computer symbolic systems such as Maple and Mathematica allow us to perform complicated and tedious calculations.

\section{Algorithm of Kudryashov method}

Let us present the algorithm of the Kudryashov method for finding exact solutions of nonlinear partial differential equations (PDE) [15]. We consider the nonlinear PDE in the following form:

$$
E\left(u, u_{t}, u_{x}, u_{t t}, u_{x x}, \ldots\right)=0 .
$$

Using the travelling wave solutions

$$
u(x, t)=y(\eta), \quad \eta=k x-w t,
$$

eq. (1) can be converted to nonlinear ordinary differential equations (ODE)

$$
E_{1}\left(y, w y_{\eta}, k y_{\eta}, w^{2} y_{\eta \eta}, k^{2} y_{\eta \eta}, \ldots\right)=0 .
$$

To find the dominant terms we substitute

$$
y(\eta)=\eta^{-p}, \quad p>0
$$

into all terms of eq. (2). Then we compare degrees of all terms in eq. (2) and choose two or more terms with the smallest degree. The maximum value of $p$ is the pole of eq. (2) and we denote it as $N$. It should be mentioned that the method can be applied when $N$ is an integer. If the value $N$ is noninteger, we have to use the transformation of solution $y(\eta)$.

We look for exact solution of (2) in the following form:

$$
y(\eta)=a_{0}+a_{1} Q(\eta)+a_{2} Q(\eta)^{2}+\cdots+a_{N} Q(\eta)^{N},
$$

where $a_{i}, i=1,2, \ldots, N$ are unknown constants and $Q(\eta)$ is the following function:

$$
Q(\eta)=\frac{1}{1+\mathrm{e}^{\eta}}
$$




\section{Travelling wave solutions}

This function satisfies to the first-order ordinary differential equation

$$
Q_{\eta}=Q^{2}-Q .
$$

Equation (6) is necessary to calculate the derivative of function $y(\eta)$. We can calculate the necessary number of derivatives of function $y$. For instance, we consider the general case when $N$ is arbitrary. Differentiating (4) with respect to $\eta$ and taking into account (6), we have

$$
\begin{aligned}
y_{\eta} & =\sum_{i=1}^{N} a_{i} i(Q-1) Q^{i}, \\
y_{\eta \eta} & =\sum_{i=1}^{N} a_{i} i\left((i+1) Q^{2}-(2 i+1) Q+i\right) Q^{i} .
\end{aligned}
$$

The highest order derivative of $y(\eta)$ can be found in [16,17]. Next, substitute expressions (4), (5) and (6) in (1). Then we collect all terms with the same powers of function $Q(z)$ and equate the resulting expression to zero. Finally, we obtain algebraic systems of equations. Solving this system, we get values for the unknown parameters.

\section{Applications to physical models}

Example 3.1. Two-dimensional sine-Gordon:

Let us demonstrate the application of Kudryashov method for finding the exact travelling wave solutions of the two-dimensional sine-Gordon equation

$$
u_{t t}-u_{x x}-u_{y y}+m^{2} \sin u=0,
$$

and Dodd-Bullough-Mikhailov equation

$$
u_{x t}+p \mathrm{e}^{u}+q \mathrm{e}^{-2 u}=0 .
$$

The two-dimensional sine-Gordon equation (8) and Dodd-Bullough-Mikhailov equation (9) have been widely applied in many fields such as solid-state physics, nonlinear optics, fluid dynamics, fluid flow, quantum field theory, electromagnetic waves and so on [7].

To look for the travelling wave solutions of eq. (8), we make transformation $v=\mathrm{e}^{i u}$, $v(x, y, t)=V(\eta), \eta=\mu(x+\alpha y+\beta t)$, and generate the reduced nonlinear ODE in the form

$$
2 \mu^{2}\left(\beta^{2}-\alpha^{2}-1\right)\left(V V^{\prime \prime}-\left(V^{\prime}\right)^{2}\right)+m^{2}\left(V^{3}-V\right)=0,
$$

where the prime denotes the differential with respect to $\eta$. The pole of eq. (10) is equal to $N=2$, then we look for the exact travelling wave solutions in the following form:

$$
V(\eta)=a_{0}+a_{1} Q+a_{2} Q^{2},
$$

where $a_{0}, a_{1}$ and $a_{2}$ are unknown constants. Substituting (11) into (10) and taking into account relations (7), we can obtain a system of algebraic equations. Solving the resulting 
system by using Maple, we find that solution of (10) exists only in the following two cases:

$$
\begin{aligned}
& \left\{a_{0}=1, a_{1}=-4, a_{2}=4, \mu=\mu, \alpha= \pm \sqrt{\beta^{2}+\frac{m^{2}}{\mu^{2}}-1}, \beta=\beta\right\}, \\
& \left\{a_{0}=-1, a_{1}=4, a_{2}=-4, \mu=\mu, \alpha= \pm \sqrt{\beta^{2}-\frac{m^{2}}{\mu^{2}}-1}, \beta=\beta\right\} .
\end{aligned}
$$

Thus, solution of eq. (8) which corresponds to (12) is given by

$$
\begin{aligned}
u_{1}(x, y, t) & =\arccos \left(\frac{1+v_{1}^{2}(x, y, t)}{2 v_{1}(x, y, t)}\right) \\
& =\arccos \left[1+2 \operatorname{csch}^{2}\left\{\mu\left(x \pm \sqrt{\left.\beta^{2}+\frac{m^{2}}{\mu^{2}}-1 y+\beta t\right)}\right)\right],\right.
\end{aligned}
$$

where

$$
\begin{aligned}
v_{1}(x, y, t)= & 1-\frac{4}{1+\exp \left\{\mu\left(x \pm \sqrt{\beta^{2}+\left(m^{2} / \mu^{2}\right)-1} y+\beta t\right)\right\}} \\
& +\frac{4}{\left[1+\exp \left\{\mu\left(x \pm \sqrt{\beta^{2}+\left(m^{2} / \mu^{2}\right)-1} y+\beta t\right)\right\}\right]^{2}} \\
= & \tanh ^{2}\left\{\frac { \mu } { 2 } \left(x \pm \sqrt{\left.\left.\beta^{2}+\frac{m^{2}}{\mu^{2}}-1 y+\beta t\right)\right\} .}\right.\right.
\end{aligned}
$$

Next, solution of eq. (8) which corresponds to (13) is given by

$$
\begin{aligned}
u_{2}(x, y, t) & =\arccos \left(\frac{1+v_{2}^{2}(x, y, t)}{2 v_{2}(x, y, t)}\right) \\
& =\arccos \left[-1-2 \operatorname{csch}^{2}\left\{\mu\left(x \pm \sqrt{\beta^{2}-\frac{m^{2}}{\mu^{2}}-1 y+\beta t}\right)\right\}\right]
\end{aligned}
$$

where

$$
\begin{aligned}
v_{2}(x, y, t)= & -1+\frac{4}{1+\exp \left\{\mu\left(x \pm \sqrt{\beta^{2}-\left(m^{2} / \mu^{2}\right)-1} y+\beta t\right)\right\}} \\
& -\frac{4}{\left[1+\exp \left\{\mu\left(x \pm \sqrt{\beta^{2}-\left(m^{2} / \mu^{2}\right)-1} y+\beta t\right)\right\}\right]^{2}} \\
= & \tanh ^{2}\left\{\frac { \mu } { 2 } \left(x \pm \sqrt{\left.\left.\beta^{2}-\frac{m^{2}}{\mu^{2}}-1 y+\beta t\right)\right\} .}\right.\right.
\end{aligned}
$$




\section{Travelling wave solutions}

More precisely, if we take $\mu=2 k$ and $\beta=\lambda$ then our solutions of (14) and (15) turn out to the solutions as expressed in [21].

Next, to find a travelling wave solution of eq. (9), we use

$$
v=\mathrm{e}^{u}, \quad v(x, t)=V(\eta), \quad \eta=\mu(x+c t) .
$$

Substituting (16) into eq. (9), we get

$$
\mu^{2} c V V^{\prime \prime}-\mu^{2} c\left(V^{\prime}\right)^{2}+p V^{3}+q=0 .
$$

The pole of eq. (17) is equal to $N=2$. Therefore, we have

$$
V(\eta)=a_{0}+a_{1} Q+a_{2} Q^{2} .
$$

Substituting (18) into (17), we can obtain a system of algebraic equations. Solving the resulting system by using Maple, the following set of solutions is obtained:

$$
\begin{gathered}
\left\{a_{0}=\left(-\frac{q}{p}\right)^{1 / 3}, a_{1}=-6\left(-\frac{q}{p}\right)^{1 / 3}, a_{2}=6\left(-\frac{q}{p}\right)^{1 / 3},\right. \\
\left.\mu=\mu, c=-\frac{3 p}{\mu^{2}}\left(-\frac{q}{p}\right)^{1 / 3}\right\} .
\end{gathered}
$$

This in turn gives the following exact travelling wave solution of eq. (9):

$$
u(x, t)=\ln \left[\left(-\frac{q}{p}\right)^{1 / 3}\left\{1-\frac{3}{1+\cosh \left\{\mu\left(x-\left(3 p / \mu^{2}\right)(-(q / p))^{1 / 3} t\right)\right\}}\right\}\right] .
$$

Example 3.2. Coupled Schrödinger-KdV equation:

A second instructive example is the coupled Schrödinger-KdV equation

$$
\begin{aligned}
& i u_{t}=u_{x x}+u v, \\
& v_{t}+6 v v_{x}+v_{x x x}=\left(|u|^{2}\right)_{x},
\end{aligned}
$$

which describes various processes in dusty plasma, such as Langmuir, dust-acoustic wave and electromagnetic waves [7]. We suppose that eq. (21) has the travelling wave solution of the form

$$
u(x, t)=\mathrm{e}^{i \theta} U(\eta), \quad v(x, t)=V(\eta), \quad \theta=\alpha x+\beta t, \quad \eta=x+c t,
$$

where $\alpha, \beta$ and $c$ are constants. Substituting eq. (22) into eq. (21), we find that $c=2 \alpha$ and $U, V$ satisfy the following coupled nonlinear ordinary differential system:

$$
\begin{aligned}
& U^{\prime \prime}+\left(\beta-\alpha^{2}\right) U+U V=0, \\
& 2 \alpha V^{\prime}+6 V V^{\prime}+V^{\prime \prime \prime}-\left(U^{2}\right)^{\prime}=0 .
\end{aligned}
$$

The pole of the coupled equations (23) are $N=2, M=2$. As a result, Kudryashov method admits the solution of (23) in the following form:

$$
\begin{aligned}
& U=a_{0}+a_{1} Q+a_{2} Q, \\
& V=b_{0}+b_{1} Q+b_{2} Q,
\end{aligned}
$$


where $a_{0}, a_{1}, a_{2}, b_{0}, b_{1}$ and $b_{2}$ are unknown constants to be determined. Substituting (24) in the reduced ODE (23) and collecting the coefficients of $Q$ yields a system of algebraic equations. Solving the resulting system by using Maple, the following sets of solution are obtained:

$$
\begin{aligned}
& \left\{a_{0}=a_{0}, a_{1}=-2 a_{0}, a_{2}=0, b_{0}=b_{0}, b_{1}=2, b_{2}=-2\right. \\
& \left.\alpha=-a_{0}^{2}-3 b_{0}-\frac{1}{2}, \beta=a_{0}^{4}+a_{0}^{2}+9 b_{0}^{2}+2 b_{0}+6 b_{0} a_{0}^{2}+\frac{1}{4}, c=2 \alpha\right\} \\
& \left\{a_{0}=0, a_{1}=\mp 6 \sqrt{2}, a_{2}= \pm 6 \sqrt{2}, b_{0}=b_{0}, b_{1}=6, b_{2}=-6\right. \\
& \left.\alpha=-3 b_{0}-\frac{1}{2}, \beta=9 b_{0}^{2}+2 b_{0}-\frac{3}{4}, c=2 \alpha\right\} \\
& \left\{a_{0}= \pm \sqrt{2}, a_{1}=\mp 6 \sqrt{2}, a_{2}= \pm 6 \sqrt{2}, b_{0}=b_{0}, b_{1}=6, b_{2}=-6\right. \\
& \left.\alpha=-3 b_{0}-\frac{5}{2}, \beta=9 b_{0}^{2}+14 b_{0}+\frac{25}{4}, c=2 \alpha\right\} .
\end{aligned}
$$

The first two sets (25) and (26) give the exact wave solutions of eq. (21) in the following form:

$$
\begin{aligned}
& u_{1}(x, t)=a_{0} \exp \left[i\left\{\left(-a_{0}^{2}-3 b_{0}-\frac{1}{2}\right) x+D_{1} t\right\}\right]\left(1-\frac{2}{1+\mathrm{e}^{x+2 \alpha t}}\right), \\
& v_{1}(x, t)=b_{0}+\frac{2}{1+\mathrm{e}^{x+2 \alpha t}}-\frac{2}{\left(1+\mathrm{e}^{x+2 \alpha t}\right)^{2}}
\end{aligned}
$$

where

$$
D_{1}=a_{0}^{4}+a_{0}^{2}+9 b_{0}^{2}+2 b_{0}+6 b_{0} a_{0}^{2}+\frac{1}{4}
$$

and

$$
\begin{aligned}
u_{2}(x, t)= & \pm 6 \sqrt{2} \exp \left[i\left\{\left(-3 b_{0}-\frac{1}{2}\right) x+\left(9 b_{0}^{2}+2 b_{0}-\frac{3}{4}\right) t\right\}\right] \\
& \times\left[-\frac{1}{1+\mathrm{e}^{x+2 \alpha t}}+\frac{1}{\left(1+\mathrm{e}^{x+2 \alpha t}\right)^{2}}\right], \\
v_{2}(x, t)= & b_{0}+\frac{6}{1+\mathrm{e}^{x+2 \alpha t}}-\frac{6}{\left(1+\mathrm{e}^{x+2 \alpha t}\right)^{2}} .
\end{aligned}
$$

Finally, the third set (27) gives the exact wave solutions as

$$
\begin{aligned}
u_{3}(x, t)= & \pm 6 \sqrt{2} \exp \left[i\left\{\left(-3 b_{0}-\frac{5}{2}\right) x+\left(9 b_{0}^{2}+14 b_{0}+\frac{25}{4}\right) t\right\}\right] \\
\times & {\left[\frac{1}{6}-\frac{1}{1+\mathrm{e}^{x+2 \alpha t}}+\frac{1}{\left(1+\mathrm{e}^{x+2 \alpha t}\right)^{2}}\right], } \\
v_{3}(x, t)= & b_{0}+\frac{6}{1+\mathrm{e}^{x+2 \alpha t}}-\frac{6}{\left(1+\mathrm{e}^{x+2 \alpha t}\right)^{2}} .
\end{aligned}
$$




\section{Travelling wave solutions}

Moreover, if we take $a_{0}=1, b_{0}=-(8+\sqrt{19}) / 18$ and $\alpha=\frac{1}{2}$ then eq. (28) provides a new travelling wave solution of the coupled Schrödinger-KdV equation (21)

$$
\begin{aligned}
& u(x, t)=\exp \left[i\left(\frac{-1+\sqrt{19}}{6} x+t\right)\right]\left(1-\frac{2}{1+\mathrm{e}^{x+t}}\right), \\
& v(x, t)=-\frac{8+\sqrt{19}}{18}+\frac{2}{1+\mathrm{e}^{x+t}}-\frac{2}{\left(1+\mathrm{e}^{x+t}\right)^{2}} .
\end{aligned}
$$

The 3D plot of solutions (31) and (32) are shown in figures 1-4. The solution (32) represents a bell-type wave solution which is shown graphically in figure 4 .

Example 3.3. Coupled nonlinear physical models:

Consider the following coupled Higgs equation [22]:

$$
\begin{aligned}
& u_{t t}-u_{x x}+|u|^{2} u-2 u v=0, \\
& v_{t t}+v_{x x}-\left(|u|^{2}\right)_{x x}=0,
\end{aligned}
$$

and coupled integrable (2+1)-dimensional nonlinear system in the following form [12]:

$$
\begin{aligned}
& i u_{t}+u_{x x}+u v=0, \\
& v_{t}+v_{y}+\left(|u|^{2}\right)_{x}=0 .
\end{aligned}
$$

First, we consider the coupled Higgs equation (33). To obtain the travelling wave solution of (33), we consider the transformation

$$
u=\mathrm{e}^{i \theta} U(\eta), \quad v=V(\eta), \quad \theta=p x+r t, \quad \eta=x+c t .
$$

Substituting (35) into (33), we have

$$
\begin{aligned}
& \left(c^{2}-1\right) U^{\prime \prime}+\left(p^{2}-r^{2}\right) U-2 U V+U^{3}=0, \\
& \left(c^{2}+1\right) V^{\prime \prime}-2\left(U^{\prime}\right)^{2}-2 U U^{\prime \prime}=0 .
\end{aligned}
$$

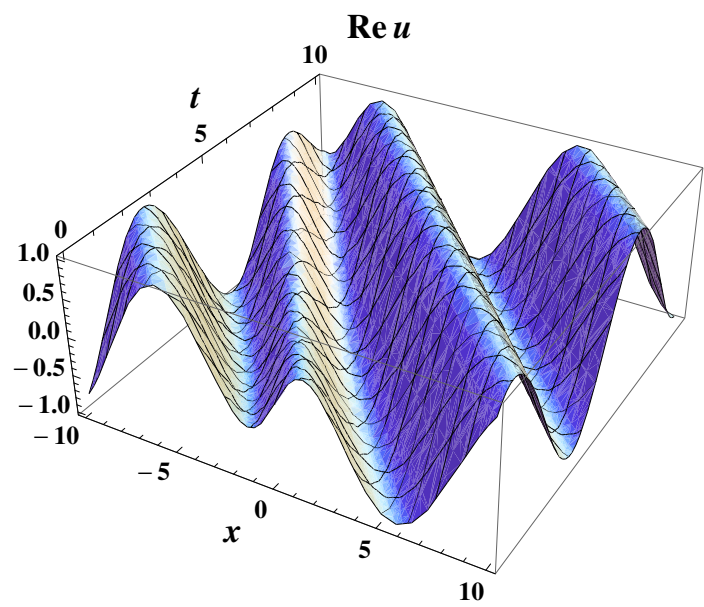

Figure 1. 3D plot of eq. (31): Real part periodic solution. 


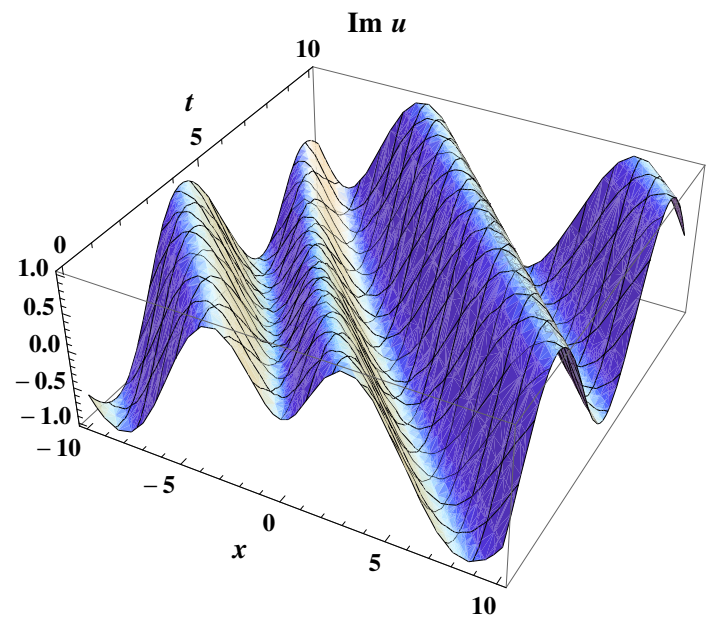

Figure 2. 3D plot of eq. (31): Imaginary part periodic solution.

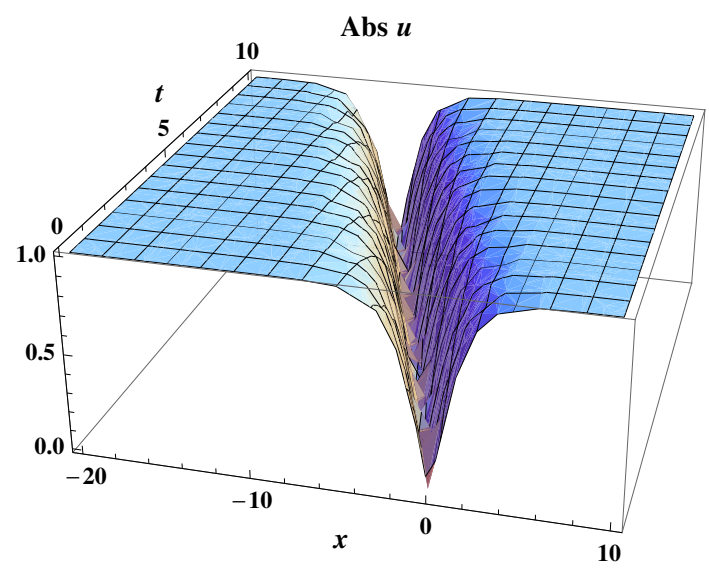

Figure 3. Kink-type wave solution of eq. (31).

Integrating the second equation in (36) and neglecting the constant of integration we find

$$
\left(c^{2}+1\right) V=U^{2} \text {. }
$$

Substituting (37) into the first equation of the system and integrating we find

$$
\left(c^{4}-1\right) U^{\prime \prime}+\left(c^{2}+1\right)\left(p^{2}-r^{2}\right) U+\left(c^{2}-1\right) U^{3}=0,
$$

where prime denotes differentiation with respect to $\eta$. The pole of eq. (38) is equal to $N=1$. Therefore, Kudryashov method admits solution in the following form:

$$
U(\eta)=a_{0}+a_{1} Q .
$$

Substituting (39) into (38), we can obtain a system of algebraic equations. Solving the resulting system with the aid of Maple, we can obtain four sets of solutions:

$$
\left\{a_{0}=a_{0}, a_{1}=a_{1}, p= \pm r, r=r, c=1\right\},
$$




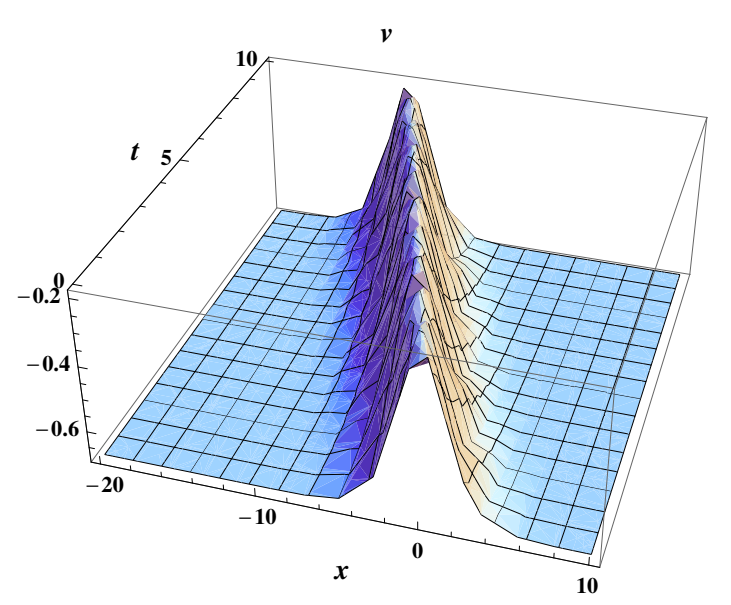

Figure 4. Bell-type wave solution of eq. (32).

$$
\begin{aligned}
& \left\{a_{0}=a_{0}, a_{1}=a_{1}, p= \pm r, r=r, c=-1\right\} \\
& \left\{a_{0}=\sqrt{-\frac{c^{2}+1}{2}}, a_{1}=-2 \sqrt{-\frac{c^{2}+1}{2}}, p= \pm \sqrt{\frac{c^{2}-1+2 r^{2}}{2}}, r=r, c=c\right\} \\
& \left\{a_{0}=-\sqrt{-\frac{c^{2}+1}{2}}, a_{1}=2 \sqrt{-\frac{c^{2}+1}{2}}, p= \pm \sqrt{\frac{c^{2}-1+2 r^{2}}{2}}, r=r, c=c\right\}
\end{aligned}
$$

According to eqs (40) and (41), we obtain the exact travelling wave solutions in the following form:

$$
u_{1}(x, t)=\mathrm{e}^{ \pm r i(x \pm t)}\left(a_{0}+\frac{a_{1}}{1+\mathrm{e}^{x+t}}\right), \quad v_{1}(x, t)=\frac{1}{c^{2}+1}\left(a_{0}+\frac{a_{1}}{1+\mathrm{e}^{x+t}}\right)^{2}
$$

and

$$
u_{2}(x, t)=\mathrm{e}^{ \pm r i(x \pm t)}\left(a_{0}+\frac{a_{1}}{1+\mathrm{e}^{x-t}}\right), \quad v_{2}(x, t)=\frac{1}{c^{2}+1}\left(a_{0}+\frac{a_{1}}{1+\mathrm{e}^{x-t}}\right)^{2} .
$$

Due to eq. (42), we get the following travelling wave solution:

$$
\begin{aligned}
& u_{3}(x, t)=\sqrt{-\frac{c^{2}+1}{2}} \exp \left[i\left( \pm \sqrt{\frac{c^{2}-1+2 r^{2}}{2}} x+r t\right)\right]\left(1-\frac{2}{1+\mathrm{e}^{x+c t}}\right) \\
& v_{3}(x, t)=-\frac{1}{2}\left(1-\frac{2}{1+\mathrm{e}^{x+c t}}\right)^{2} .
\end{aligned}
$$


Finally, eq. (43) leads to the exact travelling wave solution in the form

$$
\begin{aligned}
& u_{4}(x, t)=\sqrt{-\frac{c^{2}+1}{2}} \exp \left[i\left( \pm \sqrt{\frac{c^{2}-1+2 r^{2}}{2}} x+r t\right)\right]\left(-1+\frac{2}{1+\mathrm{e}^{x+c t}}\right) \\
& v_{4}(x, t)=-\frac{1}{2}\left(-1+\frac{2}{1+\mathrm{e}^{x+c t}}\right)^{2}
\end{aligned}
$$

In particular, if we take $c=1$ and $r=1$ then eq. (47) provides a new travelling wave solution of the coupled Higgs equation (33) in the following form:

$$
\begin{aligned}
& u(x, t)=i \exp [i(-x+t)]\left(-1+\frac{2}{1+\mathrm{e}^{x+t}}\right), \\
& v(x, t)=-\frac{1}{2}\left(-1+\frac{2}{1+\mathrm{e}^{x+t}}\right)^{2} .
\end{aligned}
$$

Further, the behaviour of the obtained solutions (48) and (49) are shown graphically (see figures 5-8).

Next, we consider the Maccari system (34). Let us assume that the travelling wave solution of (34) has the form

$$
u=\mathrm{e}^{i \theta} U(\eta), \quad v=V(\eta), \quad \theta=p x+q y+r t, \quad \eta=x+y+c t .
$$

Substituting (50) into (34), we have

$$
\begin{aligned}
& U^{\prime \prime}-\left(r+p^{2}\right) U+U V=0, \\
& (c+1) V^{\prime}-2 U U^{\prime \prime}=0 .
\end{aligned}
$$

Integrating the second equation in the system and neglecting the constant of integration we find

$$
-(c+1) V=U^{2}
$$

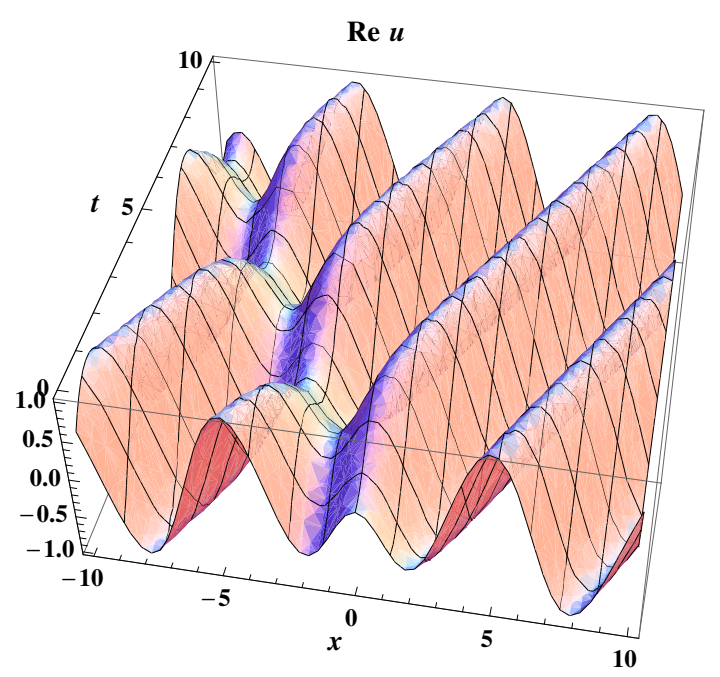

Figure 5. Real part periodic solution of eq. (48). 


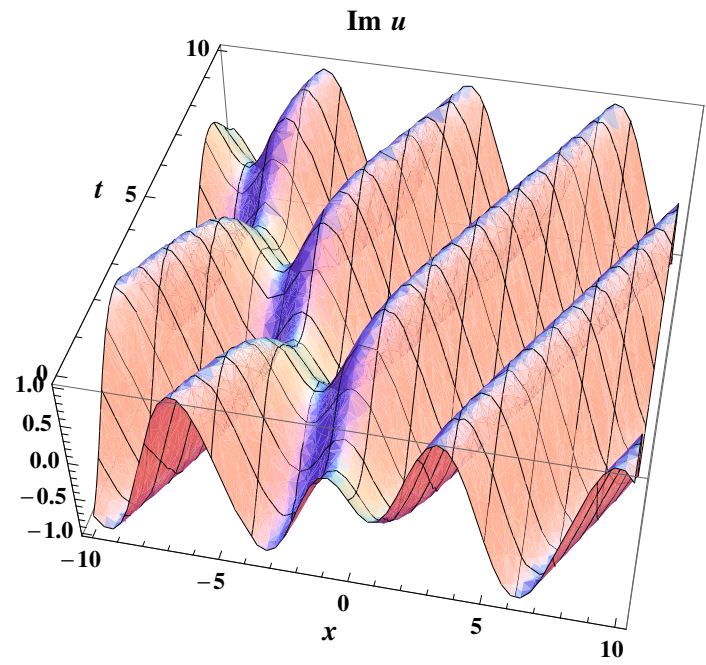

Figure 6. Imaginary part periodic solution of eq. (48).

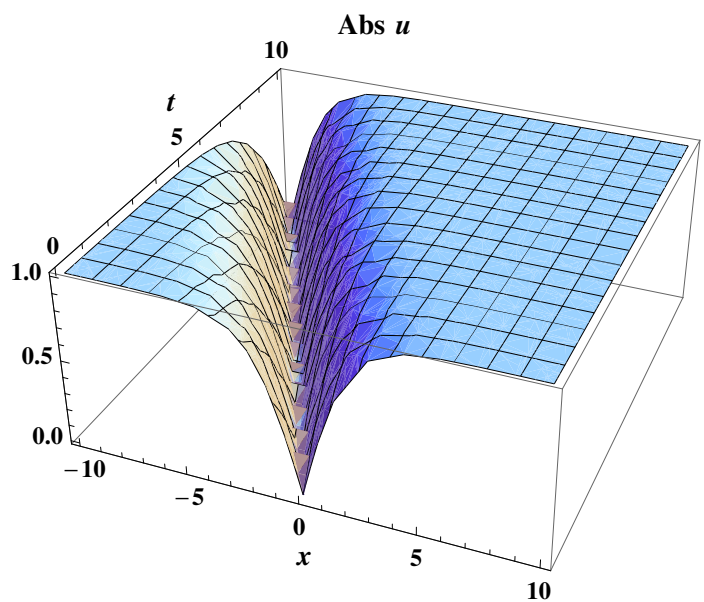

Figure 7. Kink-type wave solution of eq. (48).

Substituting (52) into the first equation of the system and integrating we find

$$
(c+1) U^{\prime \prime}-(c+1)\left(r-p^{2}\right) U-U^{3}=0,
$$

where prime denotes differentiation with respect to $\eta$. The pole of eq. (53) is equal to $N=1$. Therefore, we have

$$
U(\eta)=a_{0}+a_{1} Q .
$$

Substituting (54) into (53), we can obtain a system of algebraic equations and proceeding as before we find the following set of solution:

$$
\left\{a_{0}=-\frac{a_{1}}{2}, a_{1}=a_{1}, p=p, q=q, r=p^{2}-\frac{1}{2}, c=\frac{a_{1}^{2}}{2}-1\right\} \text {. }
$$




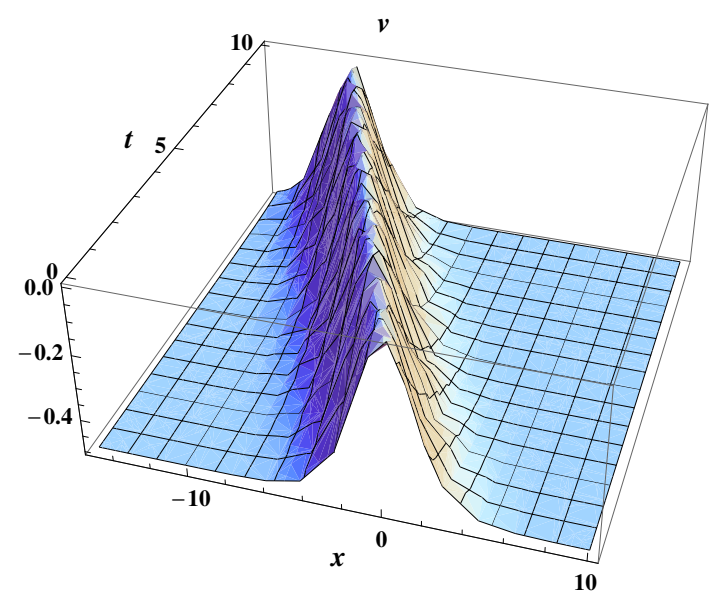

Figure 8. Bell-type wave solution of eq. (49).

The travelling wave solutions for the coupled eq. (34) according to (55) is given by

$$
\begin{aligned}
u(x, y, t)= & a_{1} \exp \left[i\left\{p x+q y+\left(p^{2}-\frac{1}{2}\right) t\right\}\right] \\
& \times\left(-\frac{1}{2}+\frac{1}{1+\exp \left[x+y+\left(\left(a_{1}^{2} / 2\right)-1\right) t\right]}\right), \\
v(x, y, t)= & -\frac{a_{1}^{2}}{c+1}\left(-\frac{1}{2}+\frac{1}{1+\exp \left[x+y+\left(\left(a_{1}^{2} / 2\right)-1\right) t\right]}\right)^{2} .
\end{aligned}
$$

Remark 3.4. When $q=0$ and $p=1$, the Dodd-Bullough-Mikhailov equation (9) reduces to the Liouville equation

$$
u_{x t}+\mathrm{e}^{u}=0
$$

By repeating the solution procedure as above, we can obtain travelling wave solution of eq. (57) in the following form:

$$
u(x, t)=\ln \left[\frac{\mu^{2} c}{1+\cosh \{\mu(x+c t)\}}\right] .
$$

Note 2.3. It is noted that Kudryashov method can be suitable to the nonlinear partial differential equations with higher order nonlinearity. It should be mentioned that all the obtained solutions are verified by putting them back into the original equations with the aid of Mathematica. 


\section{Travelling wave solutions}

\section{Conclusion}

In this paper, we have successfully implemented the Kudryashov method to establish exact solutions of the two-dimensional sine-Gordon equation and Dodd-BulloughMikhailov equation. Further, we apply the method to solve the coupled nonlinear models such as Maccari equations, Higgs equations and Schrödinger-KdV equations. The result reveals that nonlinear evolution equations can be easily handled by Kudryashov method and that the performance of this method is reliable and effective. The method is straightforward and concise, and it can also be applied to other nonlinear problems.

\section{References}

[1] J H He, Int. J. Nonlinear Mech. 34, 699 (1999)

[2] M Dehghan and R Salehi, Z. Naturforsch. A (J. Phys. Sci.) 66, 259 (2011)

[3] J H He, Comput. Method. Appl. Math. 178, 257 (1999)

[4] L Wazzan, Commun. Nonlinear Sci. Numer. Simulat. 14, 2642 (2009)

[5] J Lee and R Sakthivel, Mod. Phys. Lett. B24, 1011 (2010)

[6] A Jabbari, H Kheiri and A Bekir, Comput. Math. Appl. 62, 2177 (2011)

[7] B Salim Bahrami, H Abdollahzadeh, I M Berijani, D D Ganji and M Abdollahzadeh, Pramana - J. Phys. 77, 263 (2011)

[8] S Zhang, L Dong, J-M Ba and Y-N Sun, Pramana - J. Phys. 74, 207 (2010)

[9] M Dehghan, J Manafian and A Saadatmandi, Int. J. Mod. Phys. B25, 2965 (2011)

[10] R Sakthivel and C Chun, Z. Naturforsch. A (J. Phys. Sci.) 65a, 197 (2010)

[11] R Sakthivel, C Chun and J Lee, Z. Naturforsch. A (J. Phys. Sci.) 65, 633 (2010)

[12] S Zhang, Phys. Lett. A371, 65 (2007)

[13] Y Gurefe, A Sonmezoglu and E Misirli, Pramana - J. Phys. 77, 1023 (2011)

[14] M Dehghan and F Fakhar-Izadi, Math. Comput. Model. 53, 1865 (2011)

[15] N A Kudryashov, J. Appl. Math. Mech. 52, 361 (1988)

[16] N A Kudryashov, Commun. Nonlinear Sci. Numer. Simulat. 17, 2248 (2012)

[17] N A Kudryashov, D I Sinelshchikov and M V Demina, Phys. Lett. A375, 1074 (2011)

[18] N A Kudryashov, P N Ryabov and D I Sinelshchikov, J. Comput. Appl. Math. 235, 4513 (2011)

[19] P N Ryabov, Appl. Math. Comput. 217, 3585 (2010)

[20] M M Kabir, A Khajeh, E Abdi Aghdam and A Yousefi Koma, Math. Meth. Appl. Sci. 34, 213 (2011)

[21] E Fan and Y C Hon, Appl. Math. Comput. 141, 351 (2003)

[22] M Tajiri, J. Phys. Soc. Jpn 52, 2277 (1983) 